\title{
A GENERALIZED HYPERBOLIC METRIC FOR PLANE DOMAINS
}

\author{
LINDA KEEN AND NIKOLA LAKIC
}

\begin{abstract}
A plane domain $\Omega$ with more than one boundary point admits a hyperbolic metric and with respect to this metric, every holomorphic map of $\Omega$ into a subdomain $X \subseteq \Omega$ is a contraction. In this paper we define a new metric for the image domain $X$ that is greater than or equal to the hyperbolic metric. Like the hyperbolic metric it has the property that any holomorphic map from $\Omega$ into $X$ is a contraction. This metric has applications to random holomorphic iteration.
\end{abstract}

\section{INTRODUCTION}

The hyperbolic density $\rho(\zeta)$ on the unit disk $\Delta$ is defined for all $\zeta \in \Delta$ by the formula $\rho(\zeta)=1 /\left(1-\zeta^{2}\right)$. The hyperbolic metric is defined from this density by integration. That is,

$$
\rho\left(\zeta_{1}, \zeta_{2}\right)=\inf \int_{\gamma} \rho(\tau)|d \tau|
$$

where the infimum is taken over all paths in $\Delta$ joining $\zeta_{1}$ and $\zeta_{2}$. We use the same notation for the density and the metric; the distinction is determined by the number of parameters. The length of $\gamma$ is denoted by $\rho(\gamma)$. In this paper we will assume known standard properties of this metric such as its completeness and that the infinitesimal form of the distance is the density. Proofs of these properties and others may be found in the literature, for example [4], chap. 7.

Let $\Omega$ be an arbitrary fixed plane domain. In general we will assume that the complement of $\Omega$ in $\mathbb{C}$ contains at least two points so that any universal covering space is holomorphically equivalent to the unit disk. Such a domain is called a hyperbolic domain.

If $X$ is a hyperbolic domain and $\pi_{X}$ is a holomorphic universal covering map from the disk onto $X$, the hyperbolic density on $X$ is defined by requiring the projection map to be a local isometry:

$$
\rho_{X}(z)=\frac{\rho(\zeta)}{\left|\pi_{X}^{\prime}(\zeta)\right|} \text { for } z=\pi_{X}(\zeta) \in X
$$

It is not difficult to prove (see for example [4] chap.7) that $\rho_{X}$ is a positive continuous function.

The hyperbolic metric $\rho_{X}\left(z_{1}, z_{2}\right)$ is also defined by integrating the density over paths joining the endpoints and taking the infimum over all such paths.

2000 Mathematics Subject Classification. Primary 32G15; Secondary 30C60, 30C70, 30 C75. Paritally supported by a PSC-CUNY Grant.

Partially supported by NSF grant DMS 0200733 . 
In this paper, we will concern ourselves with given hyperbolic domains $\Omega$ and $X$ and the family of holomorphic functions from $\Omega$ into $X$ which we denote by $\mathcal{H}$ ol $(\Omega, X)$.

It follows from the Schwarz-Pick lemma that any function $f \in \mathcal{H} \operatorname{lol}(\Omega, X)$ is a contraction with respect to both $\rho_{\Omega}$ and $\rho_{X}$ considered either as a density or a metric (see theorem 1).

In this paper we generalize this situation: we define a new metric for the image domain $X$ in terms of a density that generalizes the hyperbolic density $\rho_{X}$. Distances in this new metric are greater than or equal to distances in the hyperbolic metric and it also has the property that any holomorphic map of $\Omega$ into $X$ is a contraction either in terms of the density or the metric.

When $X \subseteq \Omega$, this new metric can be used to study how the geometry of the domains $\Omega$ and $X$ affects the iterated function systems defined by functions in $\mathcal{H o l}(\Omega, X)$. This is carried out in [4], chapters 12 and 13 .

In section 2 we present a proof of theorem 1 . In section 3 we define the metric $\kappa\left(=\kappa_{X}^{\Omega}\right)$ for $X$ and describe its properties. In section 4 we give examples.

\section{The SCHWARz-PiCK LEMMA}

The classical Schwarz-Pick lemma says that any holomorphic map from the unit disk to itself is a contraction with respect to the hyperbolic metric. It generalizes to arbitrary domains using the universal holomorphic covering maps as follows.

Theorem 1 (Schwarz lemma for arbitrary domains). If $f$ is a holomorphic map from a domain $\Omega$ into a domain $X$, then $f$ is an infinitesimal and global contraction with respect to the corresponding hyperbolic metrics on $\Omega$ and $X$. That is,

$$
\rho_{X}(f(t))\left|f^{\prime}(t)\right| \leq \rho_{\Omega}(t) \text { for all } t \in \Omega
$$

and

$$
\rho_{X}\left(f(t), f\left(t^{\prime}\right)\right) \leq \rho_{\Omega}\left(t, t^{\prime}\right) \text { for all } t, t^{\prime} \in \Omega .
$$

Moreover, if $f$ is a regular covering map, it is a local isometry, that is

$$
\rho_{X}(f(t))\left|f^{\prime}(t)\right|=\rho_{\Omega}(t) \text { for all } t \in \Omega
$$

Proof. Let $\pi_{\Omega}$ and $\pi_{X}$ be the universal covering maps from the unit disk to $\Omega$ and $X$ respectively. Pick pre-images $\zeta$ and $\zeta_{f}$ in $\Delta$ of $t$ in $\Omega$ and $f(t)$ in $X$ under $\pi_{\Omega}$ and $\pi_{X}$ respectively. Now lift the map $f$ to a map $\tilde{f}$ from $\Delta$ into $\Delta$ as follows. Pick an arbitrary point $\zeta^{\prime}$ in $\Delta$. Join the point $\zeta^{\prime}$ to the point $\zeta$ by any curve $\gamma$. Project $\gamma$ down to the curve $\pi(\gamma)$ in $\Omega$ and find its image $f(\pi(\gamma))$ in $X$. Finally lift the curve $f(\pi(\gamma))$ to a curve $\tilde{\gamma}$ in $\Delta$ that starts at the point $\zeta_{f}$. The endpoint of $\tilde{\gamma}$ is by definition $\tilde{f}\left(\zeta^{\prime}\right)$. By the monodromy theorem, $\tilde{f}$ is well defined because the unit disk $\Delta$ is simply connected. Since the covering maps are holomorphic and locally one-to-one, the map $\tilde{f}$ is holomorphic. Furthermore,

$$
f \circ \pi_{\Omega}(\zeta)=\pi_{X} \circ \tilde{f}(\zeta)
$$

for all $\zeta$ in $\Delta$. Therefore, taking derivatives on both sides we obtain

$$
f^{\prime}\left(\pi_{\Omega}(\zeta)\right) \pi_{\Omega}^{\prime}(\zeta)=\pi_{X}^{\prime}(\tilde{f}(\zeta)) \tilde{f}^{\prime}(\zeta)
$$


Applying the standard Schwarz-Pick lemma for the unit disk (see for example [1]) we have

$$
\rho(\tilde{f}(\zeta))\left|\tilde{f}^{\prime}(\zeta)\right| \leq \rho(\zeta)
$$

Combining (5), (6) and (1) we obtain,

$$
\begin{gathered}
\rho(\tilde{f}(\zeta))\left|f^{\prime}\left(\pi_{\Omega}(\zeta)\right) \pi_{\Omega}^{\prime}(\zeta)\right| \leq \rho(\zeta)\left|\pi_{X}^{\prime}(\tilde{f}(\zeta))\right|, \\
\rho_{X}\left(\pi_{X}(\tilde{f}(\zeta))\right)\left|f^{\prime}\left(\pi_{\Omega}(\zeta)\right)\right| \leq \rho_{\Omega}\left(\pi_{\Omega}(\zeta)\right), \\
\rho_{X}\left(f\left(\pi_{\Omega}(\zeta)\right)\right)\left|f^{\prime}\left(\pi_{\Omega}(\zeta)\right)\right| \leq \rho_{\Omega}\left(\pi_{\Omega}(\zeta)\right) .
\end{gathered}
$$

Since $\pi_{\Omega}$ is surjective, (2) holds.

To show (3), let $t$ and $t^{\prime}$ be any two points in $\Omega$. By standard hyperbolic geometry for the unit disk, there exists a shortest path $\gamma$ in $\Omega$ joining $t$ and $t^{\prime}$. Using formula (2), we obtain,

$$
\begin{gathered}
\rho_{X}\left(f(t), f\left(t^{\prime}\right)\right) \leq \rho_{X}(f(\gamma))=\int_{f(\gamma)} \rho_{X}(\tau)|d \tau|=\int_{\gamma} \rho_{X}(f(\sigma))\left|f^{\prime}(\sigma)\right||d \sigma| \leq \\
\int_{\gamma} \rho_{\Omega}(\sigma)|d \sigma|=\rho_{\Omega}(\gamma)=\rho_{\Omega}\left(t, t^{\prime}\right) .
\end{gathered}
$$

Now suppose $f$ is a regular covering map. The composition $f \circ \pi_{\Omega}$ is a universal covering map from $\Delta$ onto $X$. Indeed, if $\gamma$ is any curve in $X$ it can be lifted to $\Delta$ first by lifting it to $\Omega$ using any inverse branch of $f$ and then by lifting the image in $\Omega$ to $\Delta$ by any inverse branch of $\pi_{\Omega}$. Now for any pre-images $t=f^{-1}(z)$ and $\zeta=\pi_{\Omega}^{-1}(z)$ the relation (1) implies

$$
\rho_{\Omega}(t)\left|\pi_{\Omega}^{\prime}(\zeta)\right|=\rho(\zeta)=\rho_{X}(f(t))\left|\left(f \circ \pi_{\Omega}\right)^{\prime}(\zeta)\right|
$$

Therefore, by the chain rule,

$$
\rho_{\Omega}(t)\left|\pi_{\Omega}^{\prime}(\zeta)\right|=\rho_{X}(f(t))\left|f^{\prime}(t)\right|\left|\pi_{\Omega}^{\prime}(\zeta)\right|,
$$

so that

$$
\rho_{\Omega}(t)=\rho_{X}(f(t))\left|f^{\prime}(t)\right|
$$

\section{KOBAyASHI METRICS}

3.1. The classical Kobayashi density. The contraction property (2) implies that any $f \in \mathcal{H o l}(\Delta, X)$, whether or not it is a covering map, satisfies

$$
\rho_{X}(f(\zeta)) \leq \frac{\rho(\zeta)}{\left|f^{\prime}(\zeta)\right|}
$$

for all points $\zeta$ in $\Delta$. Combining (1) and (7) we obtain the following formula

$$
\rho_{X}(z)=\inf \frac{\rho(\zeta)}{\left|f^{\prime}(\zeta)\right|}
$$

where the infimum is over all $f \in \mathcal{H o l}(\Delta, X)$ and all $\zeta$ in $\Delta$, such that $f(\zeta)=z$.

This leads to the Kobayashi definition of a density for an arbitrary domain $X$. 
Definition 1. The Kobayashi density $\kappa_{X}$ for the domain $X$ is defined by the formula

$$
\kappa_{X}(z)=\inf \frac{\rho(\zeta)}{\left|f^{\prime}(\zeta)\right|}
$$

where the infimum is over all $f \in \mathcal{H}$ ol $(\Delta, X)$ and all $\zeta$ in $\Delta$, such that $f(\zeta)=z$.

If $X$ is a hyperbolic domain, the universal covering map $\pi_{X}$ belongs to $\mathcal{H o l}(\Delta, X)$ and by the equation (1) realizes the infimum so that the Kobayashi density coincides with the hyperbolic density on $X$. Because any covering map $\pi_{X}$ from $\Delta$ to $X$ can be pre-composed with a conformal self map of the unit disk that maps zero to any given pre-image of the point $z$ in $X$, we may assume that $\zeta=0$ in formulas (1) and (8). Another useful formula for all hyperbolic domains $X$ is therefore

$$
\kappa_{X}(z)=\rho_{X}(z)=\frac{1}{\left|\pi^{\prime}(0)\right|}=\inf \frac{1}{\left|f^{\prime}(0)\right|}
$$

where $\pi(0)=z$ and the infimum is over all $f \in \mathcal{H}$ ol $(\Delta, X)$ such that $f(0)=z$.

Definition 1 , does not require that $X$ be a hyperbolic domain. In fact, if $X$ is either the whole plane $\mathbb{C}$, or the plane punctured at a point $p, \mathbb{C} \backslash\{p\}$, the Kobayashi density makes sense.

Theorem 2. If $X$ is either the whole plane or the whole plane punctured at a single point, then $\kappa_{X}(z)=0$ for any point $z$ in $X$.

Proof. Suppose that $X$ is either $\mathbb{C}$ or $\mathbb{C} \backslash\{p\}$. Take any point $z$ in $X$, let $N$ be a positive integer and set $f(\zeta)=p+(z-p) e^{N \zeta}$. Then $f \in \mathcal{H o l}(\Delta, X)$ and $f(0)=z$. Formula (10) implies,

$$
\kappa_{X}(z) \leq \frac{1}{\left|f^{\prime}(0)\right|}=\frac{1}{|z-p| N}
$$

Letting $N$ converge to infinity, we see that $\kappa_{X}(z)=0$.

Therefore, whenever the hyperbolic density is not defined, the Kobayashi density is identically equal to zero.

3.2. The generalized Kobayashi density. The definition of the Kobayashi density for a hyperbolic domain $X$ depends on the hyperbolic density of the unit disk $\Delta$. Any holomorphic map pushes the density on $\Delta$ forward onto $X$ and the Kobayashi density is the smallest possible push-forward. To generalize the density we change the fixed domain $\Delta$ to an arbitrary hyperbolic fixed domain $\Omega$.

Definition 2. Let $X$ be a domain in the plane. For every $z$ in $X$ let

$$
\kappa_{X}^{\Omega}(z)=\inf \frac{\rho_{\Omega}(t)}{\left|f^{\prime}(t)\right|},
$$

where $\rho_{\Omega}$ is the hyperbolic density on $\Omega$ and the infimum is over all $f \in \mathcal{H}$ ol $(\Omega, X)$ and all points $t$ in $\Omega$ such that $f(t)=z$. 
If a holomorphic map $f$ from $\Omega$ to $X$ has a critical point at $t$, as for example in the case where $f$ is a constant map, then by the continuity of $\rho_{\Omega}$ the quotient inside the infimum is a positive number divided by zero and, as is usually done in the arithmetic of the extended real line, we take it be positive infinity. Therefore, the range of $\kappa_{X}^{\Omega}$ is always contained in the interval $[0, \infty]$. We call $\kappa_{X}^{\Omega}$ the Kobayashi density on $X$ with respect to the domain $\Omega$, or simply the generalized Kobayashi density.

3.3. Properties of the generalized Kobayashi density: The first property of the generalized Kobayashi density is the following simple comparison between it and the hyperbolic density.

Proposition 1. For every hyperbolic domain $X$ and every point $z$ in $X$

$$
\rho_{X}(z) \leq \kappa_{X}^{\Omega}(z) .
$$

Proof. Let $f$ be any holomorphic map from $\Omega$ to $X$ with $f(t)=z$. Then $f$ is a weak contraction with respect to the corresponding Poincaré metrics and it follows that

$$
\begin{gathered}
\rho_{X}(z)\left|f^{\prime}(t)\right| \leq \rho_{\Omega}(t) \text { and } \\
\rho_{X}(z) \leq \frac{\rho_{\Omega}(t)}{\left|f^{\prime}(t)\right|} .
\end{gathered}
$$

Taking the infimum we get $\rho_{X}(z) \leq \kappa_{X}^{\Omega}(z)$.

Corollary 1. For all hyperbolic domains $X$ and all $z$ in $X, \kappa_{X}^{\Omega}(z)>0$.

We saw above that when $\Omega$ is the unit disk $\Delta$, the uniformization theorem implies that the holomorphic universal covering map onto the hyperbolic domain $X$ realizes the infimum in the definition of the classical Kobayashi density.

The second property of the generalized Kobayashi density is that whenever there is a regular holomorphic covering from $\Omega$ to the hyperbolic domain $X$, the generalized Kobayashi density reduces to the classical Kobayashi density.

Proposition 2. If there exists a regular holomorphic covering map from $\Omega$ onto $X$ then

In particular,

$$
\kappa_{X}^{\Omega} \equiv \rho_{X} .
$$

$$
\kappa_{\Omega}^{\Omega} \equiv \rho_{\Omega}
$$

Proof. Let $g$ be a regular holomorphic covering map from the basepoint domain $\Omega$ to the hyperbolic domain $X$. By theorem 1,

$$
\rho_{\Omega}(t)=\rho_{X}(g(t))\left|g^{\prime}(t)\right|
$$

for all $t$ in $\Omega$. For any point $z$ in $X$, since $g$ is surjective, there exists a point $t$ in $X$ such that $g(t)=z$. Now, on the one hand, by the definition of the generalized Kobayashi density, it follows that

$$
\kappa_{X}^{\Omega}(z) \leq \frac{\rho_{\Omega}(t)}{\left|g^{\prime}(t)\right|}=\rho_{X}(z)
$$

and on the other, from the first property, it follows that

$$
\kappa_{X}^{\Omega}(z) \geq \rho_{X}(z) .
$$


An immediate corollary is

Corollary 2. If $\Omega$ is a simply connected hyperbolic domain then

$$
\kappa_{X}(z)=\rho_{X}(z)=\kappa_{X}^{\Omega}(z)
$$

for all hyperbolic domains $X$ and all $z$ in $X$.

The third property is that, just as for the hyperbolic density, every holomorphic map from one domain $X$ to another domain $Y$ is an infinitesimal weak contraction with respect to the corresponding generalized Kobayashi densities.

Proposition 3. If $g \in \mathcal{H} \operatorname{lol}(X, Y)$ then

$$
\kappa_{Y}^{\Omega}(g(z))\left|g^{\prime}(z)\right| \leq \kappa_{X}^{\Omega}(z)
$$

for all $z$ in $X$.

Proof. Take $\epsilon>0$. By the definition of $\kappa_{X}^{\Omega}(z)$, there exists a point $t$ in $\Omega$ and a holomorphic map $f$ from $\Omega$ to $X$ such that $f(t)=z$ and

$$
\kappa_{X}^{\Omega}(z) \geq \frac{\rho_{\Omega}(t)}{\left|f^{\prime}(t)\right|}-\epsilon .
$$

Therefore, using the map $g \circ f$ in the definition of $\kappa_{Y}^{\Omega}(g(z))$, and applying the chain rule, it follows that

$$
\kappa_{Y}^{\Omega}(g(z))\left|g^{\prime}(z)\right| \leq \frac{\rho_{\Omega}(t)}{\left|f^{\prime}(t)\right|} \leq \epsilon+\kappa_{X}^{\Omega}(z) .
$$

Letting $\epsilon$ tend to zero, gives the required inequality.

To define the generalized Kobayashi distance between any two points in $X$ we integrate the density $\kappa_{X}^{\Omega}$.

\section{Definition 3.}

$$
\kappa_{X}^{\Omega}\left(z_{1}, z_{2}\right)=\inf \int_{\gamma} \kappa_{X}^{\Omega}(s)|d s|,
$$

where the infimum is over all paths $\gamma$ in $X$ joining $z_{1}$ to $z_{2}$.

Notice that we are again using the same notation for both the density and the distance between points where the distinction again is determined by the number of parameters.

REMARK. It may happen that the only holomorphic functions in $\mathcal{H o l}(\Omega, X)$ are constants so that $\kappa_{X}^{\Omega} \equiv \infty$ and $\kappa_{X}^{\Omega}\left(z_{1}, z_{2}\right)=\infty$ for any pair $z_{1}, z_{2} \in X$. We can interpret this as saying that $z_{1}$ and $z_{2}$ are in different components of $X$. Thus, at such points, the topology induced by the generalized Kobayashi density is not equivalent to the hyperbolic (or standard Euclidean) topology of $X$. Example 1 in section 4 shows that this can happen.

It may also happen that there are non-constant holomorphic functions from $\Omega$ to $X$, but they all have a critical value at some $z_{0} \in X$. At these points $\kappa_{X}^{\Omega}\left(z_{0}\right)$ is infinite. In Markowsky's example in section 4, there is exactly one non-constant holomorphic function from $\Omega$ to $X$ and it has a single critical value at the origin. For this example $\kappa_{X}^{\Omega}(z)<\infty$ and $\kappa_{X}^{\Omega}(0, z)<\infty$ for any non-zero $z \in X$. 
To justify our terminology we next prove that $\kappa_{X}^{\Omega}(z, w)$ is actually a complete metric on $X$ with the caveat that $\kappa_{X}^{\Omega}(z, w)$ can be infinite.

Proposition 4. $\left(X, \kappa_{X}^{\Omega}\right)$ is a complete metric space.

Proof. First we show $\kappa_{X}^{\Omega}$ is a metric. The symmetry and the triangle inequality follow directly from the definition of the distance between any two points. To show that the distance between different points is nonzero, suppose that $z_{1}$ and $z_{2}$ are any two distinct points in $X$. Choose $\epsilon>0$ and a curve $\gamma$ in $X$ that is $\epsilon$-close to realizing the infimum so that $\kappa_{X}^{\Omega}\left(z_{1}, z_{2}\right) \geq \int_{\gamma} \kappa_{X}^{\Omega}(s)|d s|-\epsilon$. Then by proposition 1 ,

$$
\kappa_{X}^{\Omega}\left(z_{1}, z_{2}\right) \geq \int_{\gamma} \rho_{X}(s)|d s|-\epsilon \geq \rho_{X}\left(z_{1}, z_{2}\right)-\epsilon,
$$

and letting $\epsilon$ tend to zero,

$$
\kappa_{X}^{\Omega}\left(z_{1}, z_{2}\right) \geq \rho_{X}\left(z_{1}, z_{2}\right)>0 .
$$

Now we show $\kappa_{X}^{\Omega}$ is complete. Suppose that $a_{n}$ is a Cauchy sequence with respect to $\kappa_{X}^{\Omega}$. Inequality (12) implies that $a_{n}$ is a Cauchy sequence with respect to $\rho_{X}$. By the completeness of $\left(X, \rho_{X}\right)$ there exists a point $a$ in $X$ such that $\rho_{X}\left(a, a_{n}\right) \rightarrow 0$ as $n \rightarrow \infty$. We would like to show that $a_{n}$ converges to $a$ in the $\kappa_{X}^{\Omega}$ metric as well.

For each $\epsilon>0$, there exists a smallest positive integer $N(\epsilon)$ such that

$$
\kappa_{X}^{\Omega}\left(a_{n}, a_{m}\right) \leq \epsilon, \text { for all } n, m \geq N(\epsilon) .
$$

Hence, taking $\epsilon_{n}=1 / 2^{n}, \quad n=1,2,3, \ldots$, there exists a subsequence $c_{n}=$ $a_{N\left(1 / 2^{n}\right)}$ of $a_{n}$ such that $\kappa_{X}^{\Omega}\left(c_{n}, c_{n+1}\right) \leq 1 / 2^{n}$. Thus there exists a path $\gamma_{n}$ that joins $c_{n}$ to $c_{n+1}$, whose length is close to realizing the distance between its endpoints, so that

$$
\kappa_{X}^{\Omega}\left(\gamma_{n}\right)=\int_{\gamma_{n}} \kappa_{X}^{\Omega}(t)|d t| \leq 1 / 2^{n-1} .
$$

By inequality (11), the hyperbolic lengths of the curves $\gamma_{n}$ converge to zero. Since the hyperbolic density is the infinitesimal form of the hyperbolic metric, it follows that the hyperbolic metric in a neighborhood of $a$ is equivalent to the Euclidean metric, and therefore that the chain $\gamma_{N}$, made of composing all paths $\gamma_{n}$ starting with $n=N$, is a path that joins $c_{N}$ to $a$ and satisfies

$$
\kappa_{X}^{\Omega}\left(\gamma_{N}\right)=\sum_{n \geq N} \kappa_{X}^{\Omega}\left(\gamma_{n}\right) \leq 1 / 2^{N-2}
$$

It follows that $\gamma_{N}$ is a path whose length goes to zero as $N$ goes to infinity. Thus $\kappa_{X}^{\Omega}\left(c_{n}, a\right)$ goes to zero and $c_{n}$ is a $\kappa_{X}^{\Omega}$-convergent subsequence with limit $a$. This, combined with inequality (13), implies that $a_{n} \kappa_{X}^{\Omega}$-converges to $a$ as claimed.

Holomorphic functions are contractions for this new metric.

Proposition 5. Every holomorphic map is a weak contraction with respect to the generalized Kobayashi metric. That is, if $f \in \mathcal{H} o l(X, Y)$ then $\kappa_{Y}^{\Omega}\left(f\left(z_{1}\right), f\left(z_{2}\right)\right) \leq$ $\kappa_{X}^{\Omega}\left(z_{1}, z_{2}\right)$.

Proof. This is a straightforward consequence of Proposition 3. 
All of the above properties of the $\kappa_{X}^{\Omega}$ density refer to the fixed domain $\Omega$. The next property tells us what happens when we fix the target domain $X$ and vary the source domain $\Omega$.

Proposition 6. (a) If there exists a regular holomorphic covering map $g$ from $\Omega$ onto $Y$, then for all domains $X$ and all points $z$ in $X$

$$
\kappa_{X}^{\Omega}(z) \leq \kappa_{X}^{Y}(z)
$$

In particular,

(b) if $\Omega$ is a simply connected hyperbolic domain, then for all hyperbolic domains $X$ and $Y$, and all points $z$ in $X$,

$$
\rho_{X}(z)=\kappa_{X}(z)=\kappa_{X}^{\Omega}(z) \leq \kappa_{X}^{Y}(z)
$$

and

(c) if $\Omega$ and $Y$ are conformally equivalent domains, then for all domains $X$ and all points $z$ in $X$,

$$
\kappa_{X}^{\Omega}(z)=\kappa_{X}^{Y}(z)
$$

Proof. Let $z$ be a point in $X$. Take a small positive constant $\epsilon$. By the definition of $\kappa_{X}^{Y}(z)$, there exists $f \in \mathcal{H o l}(Y, X)$ and a point $t$ in $Y$, such that $f(t)=z$ and

$$
\kappa_{X}^{Y}(z) \geq \frac{\rho_{Y}(t)}{\left|f^{\prime}(t)\right|}-\epsilon .
$$

Since $g$ is a surjective map, there exists a point $w$ in $\Omega$, such that $g(w)=t$. Furthermore, since $g$ is a regular holomorphic covering map, by theorem $1, g$ is an infinitesimal isometry from $\Omega$ to $Y$. Therefore,

$$
\rho_{\Omega}(w)=\rho_{Y}(t)\left|g^{\prime}(w)\right| .
$$

The definition of $\kappa_{X}^{\Omega}(z)$ and the chain rule yield

$$
\kappa_{X}^{\Omega}(z) \leq \frac{\rho_{\Omega}(w)}{\left|(f \circ g)^{\prime}(w)\right|}=\frac{\rho_{\Omega}(w)}{\left|f^{\prime}(t) g^{\prime}(w)\right|} .
$$

Combining (14), (15) and (16), we obtain

$$
\kappa_{X}^{Y}(z)+\epsilon \geq \frac{\rho_{Y}(t)}{\left|f^{\prime}(t)\right|}=\frac{\rho_{\Omega}(w)}{\left|g^{\prime}(w)\right|\left|f^{\prime}(t)\right|} \geq \kappa_{X}^{\Omega}(z) .
$$

Letting $\epsilon$ tend to zero, we obtain (a). To show part (c), observe that the conformal map from $\Omega$ to $Y$ and its inverse are both regular holomorphic covering maps. Since there exists a universal covering map from the unit disk to any hyperbolic domain, part (b) follows from corollary 2, the Riemann mapping theorem and part (a).

While the hyperbolic density is continuous, for $\kappa_{X}^{\Omega}(z)$ we have the weaker statement

Proposition 7. The density $\kappa_{X}^{\Omega}(z)$ is upper semi-continuous for every domain $X$. 
Proof. Fix $z_{0} \in X$. If $\kappa_{X}^{\Omega}\left(z_{0}\right) \neq \infty$, then given $\epsilon>0$ we can find $f_{0} \in \mathcal{H}(\Omega, X)$ and a point $w_{0} \in \Omega$ with $f_{0}\left(w_{0}\right)=z_{0}$ and $f_{0}^{\prime}\left(w_{0}\right) \neq 0$ such that

$$
\kappa_{X}^{\Omega}\left(z_{0}\right) \geq \frac{\rho_{\Omega}\left(w_{0}\right)}{\left|f_{0}^{\prime}\left(w_{0}\right)\right|}-\epsilon
$$

Let $z_{n} \in X$ be a sequence with limit $z_{0}$. Since $f_{0}^{\prime}\left(w_{0}\right) \neq 0$ we can find a local inverse $g_{0}=f_{0}^{-1}$ and a sequence $w_{n}=g_{0}\left(z_{n}\right)$ in $\Omega$ such that $w_{n} \rightarrow w_{0}$. Because $f_{0}^{\prime}$ and $\rho_{\Omega}$ are continuous functions, given $\delta>0$ we can find an $N$ such that for all $n>N$,

It follows that

$$
\kappa_{X}^{\Omega}\left(z_{n}\right) \leq \frac{\rho_{\Omega}\left(w_{n}\right)}{\left|f_{0}^{\prime}\left(w_{n}\right)\right|} \leq \frac{\rho_{\Omega}\left(w_{0}\right)}{\left|f_{0}^{\prime}\left(w_{0}\right)\right|}+\delta .
$$

$$
\limsup _{n \rightarrow \infty} \kappa_{X}^{\Omega}\left(z_{n}\right) \leq \frac{\rho_{\Omega}\left(w_{0}\right)}{\left|f_{0}^{\prime}\left(w_{0}\right)\right|}+\delta \leq \kappa_{X}^{\Omega}\left(z_{0}\right)+\epsilon+\delta
$$

Since $\epsilon$ and $\delta$ are arbitrarily small, $\kappa_{X}^{\Omega}$ is upper semi-continuous as claimed.

As an immediate corollary we have

Corollary 3. The subset of $X$ where $\kappa_{X}^{\Omega}=\infty$ is closed.

\section{EXAmples}

EXAMPLE $1 \Omega$ is the complex plane punctured at $n \geq 2$ points and $X$ is an arbitrary plane domain whose complement contains at least $n+1$ points.

Suppose that $\Omega=\mathbb{C} \backslash\left\{p_{1}, p_{2}, p_{3}, \ldots, p_{n}\right\}$ where $p_{1}, p_{2}, p_{3}, \ldots, p_{n}$ are $n \geq 2$ disjoint points and let $f: \Omega \rightarrow X$ be any holomorphic function. Then $f$ is holomorphic in a punctured neighborhood $N_{i}$ of $p_{i}$ and has a Laurent series expansion about $p_{i}$. If $p_{i}$ is an essential singularity of $f$ then $f\left(N_{i}\right)$ covers every point in $\hat{\mathbb{C}}$, with the exception of at most two points, infinitely often. Since the complement of $X$ contains at least 2 points, $f\left(N_{i}\right)$ must cover $X$ and at least one of its boundary points, contradicting the assumption that $f(\Omega) \subset X$.

Therefore, for each $i, p_{i}$ must be either a pole or a removable singularity and $\left.f\right|_{N_{i}}$ can be extended to $p_{i}$ so that $\tilde{f}\left(p_{i}\right) \in \hat{\mathbb{C}}$ is meromorphic. Extending at each point we obtain $\tilde{f}$, whose image is compact. Since a holomorphic map is either constant or open the image is either a single point or the whole sphere with the property $f=\left.\tilde{f}\right|_{\Omega}$. Now if $f$ is not a constant function,

$$
X \supset f(\Omega)=\tilde{f}(\Omega) \supset \tilde{f}(\mathbb{C}) \backslash \cup_{i=1}^{n} \tilde{f}\left(p_{i}\right)
$$

But this cannot be since the complement of $X$ contains at least $n+1$ points. We conclude that the only holomorphic functions $f: \Omega \rightarrow X$ are constants and that $\kappa_{X}^{\Omega} \equiv \infty$.

EXAMPLE $2 \quad \Omega$ is an arbitrary hyperbolic domain and $X$ is a simply connected hyperbolic domain.

Since $X$ is a simply connected hyperbolic domain there is a biholomorphic map $h: \Delta \rightarrow X$. Applying Proposition 3 to both $h$ and $h^{-1}$ we deduce $\kappa_{X}^{\Omega}(h(z))\left|h^{\prime}(z)\right|=\kappa_{\Delta}^{\Omega}(z)$ so we may assume $X$ is $\Delta$. 
To evaluate $\kappa_{\Delta}^{\Omega}(z)$, recall that the Möbius transformation, $A(w)=\frac{w-z}{1-w \bar{z}}$ maps $z$ to 0 . Applying proposition 3 again,

$$
\kappa_{\Delta}^{\Omega}(z)=\kappa_{\Delta}^{\Omega}(0)\left|A^{\prime}(z)\right|=\frac{\kappa_{\Delta}^{\Omega}(0)}{1-|z|^{2}}=\kappa_{\Delta}^{\Omega}(0) \rho(z) .
$$

We conclude $\kappa_{X}^{\Omega}(z)=K(\Omega) \rho_{X}(z)$ where $K(\Omega)$ is a constant depending on the domain. By proposition $1, K(\Omega) \geq 1$. We saw in the previous example that $K(\Omega)$ can be infinity. We will see in Examples 3 and 4 that it can take the value one. For an example where it is finite but strictly greater than one see example 4 in [4] section 10.5 .

EXAMPLE $3 \Omega$ is a hyperbolic simply connected domain with a puncture and $X$ is an arbitrary hyperbolic domain.

Since there is a biholomorphic map from $\Omega$ union its puncture to $\Delta$, such that the puncture maps to the origin, we may, by proposition $6(\mathrm{c})$, assume $\Omega=\Delta \backslash\{0\}=$ $\Delta^{*}$. Thus we want to find $\kappa_{X}^{\Delta^{*}}(z)$ for any $z \in X$.

Fix $z \in X$ and let $\pi: \Delta \rightarrow X$ be a universal covering map. Since it is a covering, $\rho_{X}(\pi(t))\left|\pi^{\prime}(t)\right|=\rho_{\Delta}(t)$ for all $t \in \Delta$. For the moment fix $t$ such that $\pi(t)=z$.

Choosing $\left.\pi\right|_{\Delta^{*}}$ as a candidate $f$ in our definition, we have

$$
\kappa_{X}^{\Delta^{*}}(z) \leq \frac{\rho_{\Delta^{*}}(t)}{\left|\pi^{\prime}(t)\right|}=\frac{\rho_{\Delta^{*}}(t)}{\rho_{\Delta}(t)} \rho_{X}(z) .
$$

Now suppose $t_{n} \in \Delta$ with $\left|t_{n}\right| \rightarrow 1$. By pre-composing with a Möbius transformation we can choose $\pi_{n}: \Delta \rightarrow X$ such that $\pi_{n}\left(t_{n}\right)=z$. From the above we have

$$
\kappa_{X}^{\Delta^{*}}(z) \leq \frac{\rho_{\Delta^{*}}\left(t_{n}\right)}{\rho_{\Delta}\left(t_{n}\right)} \rho_{X}(z)
$$

Using the fact that $\rho_{\Delta^{*}}(t)=\frac{1}{2|t| \log \frac{1}{|t|}}$, it is an exercise to show that that

$$
\frac{\rho_{\Delta *}\left(t_{n}\right)}{\rho_{\Delta}\left(t_{n}\right)} \rightarrow 1 \text {. }
$$

We conclude $\kappa_{X}^{\Delta^{*}}(z) \leq \rho_{X}(z)$. Combining this with proposition 1 we see that $\kappa_{X}^{\Delta^{*}}(z)=\rho_{X}(z)$. In particular, $K\left(\Delta^{*}\right)=1$.

EXAMPLE 4 Let $A=\{z: 0<a<|z|<1\}$.

Let $t$ be any point in $A$ and let $t_{n}$ be any preimage of $t$ under the map $f_{n}(z)=z^{n}$. Since $f_{n}$ maps $A$ into $\Delta$, by definition,

$$
k_{\Delta}^{A}(t) \leq \frac{\rho_{A}\left(t_{n}\right)}{\left|f_{n}^{\prime}\left(t_{n}\right)\right|} .
$$

Since $f_{n}$ is a covering map from $\Delta^{*}$ onto itself, we have by theorem 1 ,

$$
\rho_{\Delta^{*}}\left(t_{n}\right)=\rho_{\Delta^{*}}(t)\left|f_{n}^{\prime}\left(t_{n}\right)\right| \text {. }
$$

By the relation 17 ,

$$
k_{\Delta}^{A}(t)=k_{\Delta}^{A}(0) \rho(t)
$$


Thus

$$
\begin{gathered}
K(A)=k_{\Delta}^{A}(0)=\frac{k_{\Delta}^{A}(t)}{\rho(t)} \leq \frac{\rho_{A}\left(t_{n}\right)}{\rho(t)\left|f_{n}^{\prime}\left(t_{n}\right)\right|}= \\
\frac{\rho_{A}\left(t_{n}\right) \rho_{\Delta^{*}}(t)}{\rho(t) \rho_{\Delta^{*}}\left(t_{n}\right)} \rightarrow \frac{\rho_{\Delta^{*}}(t)}{\rho(t)} \text { as } n \rightarrow \infty .
\end{gathered}
$$

Now letting $|t| \rightarrow 1$ it follows that $K(A) \leq 1$.

EXAMPLE 5 We conclude with an example originally worked out by Markowsky [6] that shows that the generalized Kobayashi metric can degenerate at points without being degenerate on the whole domain. In this example the Kobayashi density takes the value infinity at an isolated point, but the distance from that point to any other is still finite. The point is thus like a pole for the density function.

Let $K=\left\{\infty, e^{\frac{\pi i}{6}}, e^{\frac{\pi i}{3}}, e^{\frac{\pi i}{2}}, e^{\pi i}\right\}$ and set $\Omega=\hat{\mathbb{C}} \backslash f^{-1}(K)$. Let $f(z)=z^{2}$ and set $X=\hat{\mathbb{C}} \backslash K$; then $f$ maps $\Omega$ onto $X$.

Suppose $g$ is any non-constant holomorphic map in $\mathcal{H o l}(\Omega, X)$. Because $X$ is the complement of the Riemann sphere punctured at $n \geq 3$ points, $g$ has removable singularities at the punctures. It therefore extends to a rational function of degree $d$, also called $g$, from the sphere to itself. By the Riemann-Hurwitz formula, the cardinality of the set $g^{-1}(K) \subset f^{-1}(K)$ is at least $5 d-(2 d-2)$. Since the cardinality of $f^{-1}(K)$ is 9 , we see that $d \leq 2$.

Note that there must be at least three points $a, b, c$ in $K \backslash\{\infty\}$ whose preimages lie in $f^{-1}(K) \backslash\{\infty\}$ and hence on the unit circle.

If $d=1, g$ is a Möbius transformation that sends three points on the unit circle to $a, b, c$ and so maps the whole circle to itself. The preimage of infinity is therefore not on the unit circle so $g$ fixes infinity and must be of the form $g(z)=\lambda z,|\lambda|=1$. Since $X \nsubseteq \lambda \Omega$ we conclude $d=2$.

Let $h$ be a Möbius transformation that takes the unit circle to the real line sending $a, b, c$ to $0,1, \infty$ and set $\tilde{g}=h \circ g \circ h^{-1}$. Then $\tilde{g}$ is a rational function of degree 2 of the form

$$
\tilde{g}(z)=\lambda \frac{(z-r)(z-s)}{(z-t)(z-u)}
$$

with the usual conventions if one of the constants is infinity. Since $\tilde{g}^{-1}(\{0,1, \infty\}) \subset$ $\mathbb{R} \cup\{\infty\}$ we deduce that all the constants $\lambda, r, s, t, u$ are real and $g$ is a Blaschke product. Since $g^{-1}(K) \backslash\{\infty\}$ is contained in the unit circle, $g$ fixes infinity and is of the form $\lambda z^{2}$ with $|\lambda|=1$. Again, the only way $g(\Omega) \subseteq X$ is $\lambda=1$ so that $g(z)=z^{2}$.

Now we can easily compute the generalized Kobayashi density in terms of $\rho_{\Omega}$. We have

$$
\kappa_{X}^{\Omega}(z)=\inf _{ \pm \sqrt{z}} \frac{\rho_{\Omega}(\sqrt{z})}{|2 \sqrt{z}|} .
$$

This takes the value infinity at the origin, but nowhere else in $X$. Note that if $\gamma(t)$ is a straight segment from a point $z_{0} \in X$ that ends at the origin, its length is finite. 
This construction can easily be extended to domains $\Omega$ and $X$ with the single non-constant holomorphic map $z^{d}, d>2$ between them by removing more points from the circle and placing them appropriately.

\section{REFERENCES}

[1] L. Ahlfors, Lectures on Quasiconformal Mappings, Van Nostrand Studies 10 (1966)

[2] S. Dineen, The Schwarz lemma, Oxford Mathematical Monographs. Oxford Science Publications. The Clarendon Press, Oxford University Press, New York, 1989.

[3] F. P. Gardiner and N. Lakic, Comparing Poincaré distances Annals of Math., 154, 2001, $245-267$

[4] L. Keen and N. Lakic Hyperbolic Geometry from a local viewpoint Cambridge Univ. Press, to appear.

[5] S. G. Krantz, Complex Analysis: a geometric viewpoint, Carus Math. Monographs, 23, MAA, 1990

[6] G. Markowsky, Personal communication.

Department of Mathematics, Lehman College and Graduate Center, Cuny, Bronx NY 10468

E-mail address: linda.keen@lehman.cuny.edu

Department of Mathematics, Lehman College and Graduate Center, CUny, Bronx NY 10468

E-mail address: nikola.lakic@lehman.cuny.edu 